Y. Yoshino

Nagoya Math. J.

Vol. 81 (1981), 105-112

\title{
THE CANONICAL MODULES OF GRADED RINGS DEFINED BY GENERIC MATRICES
}

\author{
YUJI YOSHINO
}

Let $k$ be a field, and $X=\left[x_{i j}\right]$ be an $n \times(n+m)$ matrix whose elements are algebraically independent over $k$.

We shall study the canonical module of the graded ring $R$, which is a quotient ring of the polynomial ring $A=k[X]$ by the ideal $\mathfrak{a}_{n}(X)$ generated by all the $n \times n$ minors of $X$.

Nowadays it is not difficult to describe the free resolution of the canonical module of $R$, since the resolution of $R$ as an $A$ module is already known [Eagon-Northcott]. But we will be at a loss for an answer when we are asked what are the generators of the ideal of $R$ which is isomorphic to the canonical module of $R$, for in most cases the canonical modules of Cohen-Macaulay rings are isomorphic to their ideals, which we might call the canonical ideals.

For this reason, in this paper we shall write down the generators of the canonical ideal of $R$.

\section{§1. The canonical modules of graded rings}

In this section by a graded ring we understand a commutative ring $R$ with a family $\left\{R_{n}\right\}_{n \geqslant 0}$ such that;

(i) $R=\oplus R_{n}, R_{n} \cdot R_{m} \subset R_{n+m}$ for all $n, m \geqslant 0$.

(ii) $R_{0}=k$ is a field.

(iii) $R$ is finitely generated over $k$.

Here we shall recall some fundamental facts about the canonical modules of graded rings, all of which are based on the paper of GotoWatanabe [G-W].

Notation from [G-W]: For any graded ring $R$, we denote by $M_{H}(R)$ the category of all the graded $R$-modules and their homomorphisms of degree 0. Moreover we use the following symbols:

Received June 21, 1979. 
for any $M, N \in M_{H}(R)$,

$\underline{\operatorname{Hom}}_{R}(M, N)_{n}=\left\{\begin{array}{l}f: M \longrightarrow N ; R \text {-homomorphism such that } \\ f\left(M_{m}\right) \subset N_{n+m} \text { for any } m .\end{array}\right\}$

$\underline{\operatorname{Hom}}_{R}(M, N)=\oplus \underline{\operatorname{Hom}}_{R}(M, N)_{n}$

$\underline{\operatorname{Ext}}_{R}(M, N)$ are the derived functors of $\operatorname{Hom}_{R}(M, N)$ in $M_{H}(R)$,

$\underline{H}_{\mathrm{m}}^{i}(M)=\underset{v}{\lim } \underset{\operatorname{Ext}_{R}^{i}}{\operatorname{lom}}\left(R / \mathrm{m}^{v}, M\right)$ is the $i$-th local cohomology of $M$ with support in $\mathfrak{m}$, where $\mathfrak{m}=\bigoplus_{n>0} R_{n}$,

$\underline{K}_{R}=\underline{H}_{m}^{d}(R)^{*}$ is the canonical module of $R$ where ()$^{*}=\underline{\operatorname{Hom}}_{R}(, k)$, $d=\operatorname{dim} R$.

Lemma 1. Let $R=\oplus_{n \geq 0} R_{n}$ be a graded Cohen-Macaulay domain and $\mathfrak{m}=\oplus_{n>0} R_{n}$. Then,

(1) $\left(\underline{K}_{R}\right)_{\mathrm{m}}$ is the canonical module of the local ring $R_{\mathrm{m}}$ in the sense of Herzog-Kunz,

(2) $\underline{K}_{R_{-}}{ }^{i}$ is isomorphic to a homogeneous fractional ideal of $R$.

Proof. (1) From [G-W] Proposition (2.1.8) $\left(\underline{K}_{R}\right)_{\mathrm{m}}$ must be a maximal Cohen-Macaulay module over $R_{\mathrm{m}}$, and

$$
\operatorname{dim}_{k} \operatorname{Ext}_{R_{\mathrm{m}}}^{d}\left(k,\left(\underline{K}_{R}\right)_{\mathrm{m}}\right)=1 \quad \text { where } d=\operatorname{dim} R .
$$

So the statement (1) follows from [H-K] Korollar 6.12.

(2) Let $Q$ be the field of fractions of $R$. From (1) we have $\left(\underline{K}_{R}\right)_{\mathrm{m}} \otimes_{R_{\mathrm{m}}} Q$ $=Q$, so $\operatorname{dim}_{Q} \operatorname{Hom}_{Q}\left(Q, \underline{K}_{R} \otimes_{R} Q\right)=1$.

From [G-W] Theorem (1.2.4) this shows that the injective envelope $\underline{E}_{R}\left(\underline{K}_{R}\right)$ of $\underline{K}_{R}$ in $M_{H}(R)$ is isomorphic to $\left[\underline{E}_{R}(R)\right](n)$ for some integer $n$. However obviously $\underline{E}_{R}(R) \cong S^{-1} R$ where $S=$ non-zero homogeneous elements of $R$. So $\underline{K}_{R} G\left[S^{-1} R\right](n)$ in $M_{H}(R)$.

Lemma 2. Let $R=\oplus_{n \geq 0} R_{n}$ be a graded Cohen-Macaulay ring of dimension $d \geq 1$, and suppose that $\underline{K}_{R}$ is isomorphic to a homogeneous ideal $I$ of $R$. Then $I$ is of pure height 1 , and the graded ring $R / I$ is Gorenstein.

Proof. From the exact sequence

$$
0 \longrightarrow I \longrightarrow R \longrightarrow R / I \longrightarrow 0
$$

$\underline{H}_{\mathrm{m}}^{i}(R / I) \cong \underline{H}_{\mathrm{m}}^{i+1}(I) \cong \underline{H}_{\mathrm{m}}^{i+1}\left(\underline{K}_{R}\right)=0$ for $0 \leq i \leq d-2$, because $\underline{K}_{R}$ is a maximal Cohen-Macaulay module. This shows

$$
d-1 \leq \operatorname{depth} R / I \leq \inf _{\mathfrak{p} \in \operatorname{Ass} R / I} \operatorname{dim} R / \mathfrak{p}
$$


and so $h t(\mathfrak{p}) \leq 1$ for any $\mathfrak{p} \in$ Ass $R / I$.

Next the short sequence $(*)$ yields the following exact sequence

$$
\underline{\operatorname{Hom}}_{R}(R, I) \longrightarrow \underline{\operatorname{Hom}}_{R}(I, I) \longrightarrow \operatorname{Ext}_{R}^{1}(R / I, I) \longrightarrow 0 \text {. }
$$

Here by virtue of $h t(I)=1$ and [G-W] Proposition (2.2.9.)

$$
\underline{\operatorname{Ext}}_{R}^{1}(R / I, I) \cong \underline{\operatorname{Ext}}_{R}^{1}\left(R / I, \underline{K}_{R}\right) \cong \underline{K}_{R / I}
$$

and from the local duality [G-W] Proposition (2.1.6.)

$$
\underline{\operatorname{Hom}}_{R}(I, I) \cong \underline{\operatorname{Hom}}_{R}\left(\underline{K}_{R}, \underline{K}_{R}\right) \cong \underline{H}_{\mathrm{m}}^{d}\left(\underline{K}_{R}\right)^{*} \cong R^{* *} \cong R .
$$

From these facts we find that the $\underline{K}_{R / I}$ is a cyclic $R / I$ module, so by [G-W] Proposition (2.1.3.) $R / I$ is a Gorenstein ring as stated.

(Q.E.D.)

\section{$\S 2$. The structure of $\underline{K}_{R}$}

Now we shall return to the subject of this paper. And for the rest of this section we assume the following notations,

$n \geq 1, m \geq 0 ;$ fixed integers

$k$; a field

$X=\left[x_{i j}\right] ;$ an $n \times(n+m)$ matrix of indeterminates

$A=k[X] ;$ a polynomial ring in $n(n+m)$ indeterminates

$\mathfrak{a}=\mathfrak{a}_{n}(X)$; the ideal of $A$ generated by all the $n \times n$ minors of $X$

$R=A / \mathfrak{a}$

Lemma 3. $a$ is a prime ideal of height $(m+1)$ and $R$ is a CohenMacaulay ring.

Proof. [H-E].

Lemma 4 [Eagon-Northcott]. Let $K$ be the exterior algebra over $A$ generated by $\theta_{1}, \cdots, \theta_{n+m}$, and $\Delta_{j}$ the differentiation on $K$ determined by the $j$-th row of $X$, i.e.,

$$
\Delta_{j}\left(\theta_{i_{\mathbf{1}}} \wedge \cdots \wedge \theta_{i_{t}}\right)=\sum_{p=1}^{t}(-1)^{p+1} x_{j i_{p}}\left(\theta_{i_{\mathbf{1}}} \wedge \cdots \wedge \hat{\theta}_{i_{p}} \wedge \cdots \wedge \theta_{i_{\ell}}\right) .
$$

Next let $\tau_{1} \cdots \tau_{n}$ be new $n$ symbols and, in the polynomial ring $A\left[\tau_{1} \cdots \tau_{n}\right]$, denote by $\Phi_{t}$ the A-module consisting of all the forms of degree $t$.

Now we define the complex

$$
A_{.}^{X}: 0 \longrightarrow A_{m+1}^{X} \stackrel{d_{m}}{\longrightarrow} A_{m}^{X} \stackrel{d_{m-1}}{\longrightarrow} \cdots \stackrel{d_{1}}{\longrightarrow} A_{1}^{X} \stackrel{d_{0}}{\longrightarrow} A_{0}^{X} \longrightarrow 0
$$


as follows,

$$
\begin{aligned}
& A_{0}^{X}=A \\
& A_{q+1}^{X}=K_{n+q} \otimes \Phi_{q} \quad(q=0,1,2, \cdots, m) .
\end{aligned}
$$

When $q>0, d_{q}$ is given by the formula

$$
\begin{aligned}
d_{q}\left(\theta_{i_{1}}\right. & \left.\wedge \cdots \wedge \theta_{i_{n+q}} \otimes \tau_{1}^{v_{1}} \cdots \tau_{n}^{v_{n}}\right) \\
\quad & =\sum_{j}^{*} \Delta_{j}\left(\theta_{i_{1}} \wedge \cdots \wedge \theta_{i_{n+q}}\right) \otimes \tau_{1}^{v_{1}} \cdots \tau_{j}^{v_{j}-1} \cdots \tau_{n}^{v_{n}}
\end{aligned}
$$

where $\sum_{j=1}^{n} v_{j}=q$, and $\sum_{j}^{*}$ means that we sum over only those values of $j$ for which $v_{j}>0$.

For $q=0$,

$$
d_{0}\left(\theta_{i_{1}} \wedge \cdots \wedge \theta_{i_{n}}\right)=\operatorname{det}\left[\begin{array}{ccc}
x_{1 i_{1}} & \cdots & x_{1 i_{n}} \\
\vdots & & \vdots \\
x_{n i_{1}} & \cdots & x_{n i_{n}}
\end{array}\right]
$$

Moreover we put

$$
\operatorname{deg}\left(\theta_{i_{1}} \wedge \cdots \wedge \theta_{i_{n+q}} \otimes \tau_{n}^{v_{n}}\right)=n+q .
$$

Then the theorem of Eagon-Northcott says that the complex $A^{X}$ is acyclic and $H_{0}\left(A^{X}\right)=A / \mathfrak{a}=R$.

THEOREM. In addition to the notation as before we put

$$
Y=\left[\begin{array}{ccc}
x_{11} & \cdots & x_{1, n-1} \\
\vdots & & \vdots \\
x_{n 1} & \cdots & x_{n, n-1}
\end{array}\right]
$$

and $\mathfrak{p}=\mathfrak{a}_{n-1}(Y) \cdot R$ the ideal of $R$ generated by all the $(n-1) \times(n-1)$ minors of $Y$.

Then the canonical module of $R$ is isomorphic to $\mathfrak{p}^{m}(-n(n-1))$.

Proof. If we apply the functor $\operatorname{Hom}_{A}\left(, \underline{K}_{A}\right)$ to the free resolution $A^{X} \rightarrow R \rightarrow 0$ of $R$ as an $A$-module obtained in Lemma 4, we get the exact sequence,

$$
\underline{\operatorname{Hom}}_{A}\left(A_{m}^{X}, \underline{K}_{A}\right) \longrightarrow \underline{\operatorname{Hom}}_{A}\left(A_{m+1}^{X}, \underline{K}_{A}\right) \longrightarrow \underline{K}_{R} \longrightarrow 0
$$

by virtue of [G-W] Proposition (2.2.9). Here since $\underline{K}_{A}=A(-n(n+m))$ ([G-W] Cor. (2.2.8)), the sequence;

$$
\underline{\operatorname{Hom}}_{A}\left(A_{m}^{X}, A\right) \underset{d_{m}^{*}}{\longrightarrow} \underline{\operatorname{Hom}}_{A}\left(A_{m+1}^{X}, A\right) \longrightarrow \underline{K}_{R}(n(n+m)) \longrightarrow 0
$$


is exact.

For simplicity we make the following definitions,

for $v_{1}+\cdots+v_{n}=m$,

$$
\alpha\left(v_{1}, \cdots, v_{n}\right)=\left(\theta_{1} \wedge \cdots \wedge \theta_{n+m}\right) \otimes \tau_{1}^{v_{1}} \cdots \tau_{n}^{v_{n}} \in A_{m+1}^{X}
$$

and for $w_{1}+\cdots+w_{n}=m-1,1 \leq j \leq n+m$,

$$
\beta\left(j ; w_{1}, \cdots, w_{n}\right)=\theta_{1} \wedge \cdots \wedge \check{\theta}_{j} \wedge \cdots \wedge \theta_{n+m} \otimes \tau_{1}^{w_{1}} \cdots \tau_{n}^{w_{n}} \in A_{m}^{X}
$$

where if there exists $i$ such that $v_{i}<0$ (resp. $w_{i}<0$ ) then $\alpha\left(v_{1}, \cdots, v_{n}\right.$ ) $=0\left(\right.$ resp. $\left.\beta\left(j ; w_{1}, \cdots, w_{n}\right)=0\right)$.

Then as we have seen in Lemma 4 ,

$$
\left\{\alpha\left(v_{1}, \cdots, v_{n}\right) / \sum_{i=1}^{n} v_{i}=m\right\}\left\{\beta\left(j ; w_{1}, \cdots, w_{n}\right) / \sum_{i=1}^{n} w_{i}=m-1,1 \leq j \leq n+m\right\}
$$

are the free bases of $A_{m+1}^{X}, A_{m}^{X}$ respectively, and from the definition,

$$
d_{m}\left(\alpha\left(v_{1}, \cdots, v_{n}\right)\right)=\sum_{q=1}^{n} \sum_{p=1}^{n+m}(-1)^{p} x_{q p} \beta\left(p ; v_{1}, \cdots, v_{q}-1, \cdots, v_{n}\right)
$$

for every $\left(v_{1}, \cdots, v_{n}\right)$ such that $\sum_{i=1}^{n} v_{i}=m$.

Next we define $\alpha^{*}\left(v_{1}, \cdots, v_{n}\right) \in \underline{\operatorname{Hom}}_{A}\left(A_{m+1}^{X}, A\right)$ to be $\alpha^{*}\left(v_{1}, \cdots, v_{n}\right)$. $\alpha\left(v_{1}^{\prime}, \cdots, v_{n}^{\prime}\right)=\delta_{v_{1} v_{1}^{\prime}} \cdots \delta_{v_{n} v_{n}^{\prime}}$. Then $\left\{\alpha^{*}\left(v_{1}, \cdots, v_{n}\right) / \sum v_{i}=m\right\}$ is a free base of $\operatorname{Hom}_{A}\left(A_{m+1}^{X}, A\right)$ and $\operatorname{deg} \alpha^{*}\left(v_{1}, \cdots, v_{n}\right)=-(n+m)$. In the same way a free base $\left\{\beta^{*}\left(j ; w_{1}, \cdots, w_{n}\right) / \sum_{i=1}^{n} w_{i}=m-1,1 \leq j \leq n+m\right\}$ of $\underline{\operatorname{Hom}}_{A}\left(A_{m}^{X}, A\right)$ is defined by

$$
\beta^{*}\left(j ; w_{1}, \cdots, w_{n}\right) \cdot \beta\left(j^{\prime} ; w_{1}^{\prime}, \cdots, w_{n}^{\prime}\right)=\delta_{j j^{\prime}} \cdot \delta_{w_{1} w_{1}^{\prime}} \cdots \delta_{w_{n} w_{n}^{\prime}}
$$

and so $\operatorname{deg} \beta^{*}\left(j ; w_{1}, \cdots, w_{n}\right)=-(n+m-1)$.

In consequence if $1 \leq j \leq n+m, \sum_{i=1}^{n} w_{i}=m-1, \sum_{i=1}^{n} v_{i}=m$, then

$$
\begin{aligned}
d_{m}^{*}\left(\beta^{*}\right. & \left.\left(j ; w_{1}, \cdots, w_{n}\right)\right) \cdot \alpha\left(v_{1}, \cdots, v_{n}\right) \\
& =\beta^{*}\left(j ; w_{1}, \cdots, w_{n}\right) \cdot d_{m}\left(\alpha\left(v_{1}, \cdots, v_{n}\right)\right) \\
& =\left\{\begin{array}{cl}
(-1)^{j+1} x_{q j} & \text { when }\left(v_{1}, \cdots, v_{q}-1, \cdots, v_{n}\right)=\left(w_{1}, \cdots, w_{n}\right) \\
0 & \text { otherwise } .
\end{array}\right.
\end{aligned}
$$

This shows that

$$
d_{m}^{*}\left(\beta^{*}\left(j ; w_{1}, \cdots, w_{n}\right)\right)=(-1)^{j+1} \sum_{q=1}^{n} x_{q j} \alpha^{*}\left(w_{1}, \cdots, w_{q}+1, \cdots, w_{n}\right) .
$$


Now let $Y_{i}$ denote the matrix obtained by deleting the $i$-th row of $Y$ and suppose

$$
\delta_{i}=(-1)^{i+1} \operatorname{det} Y_{i}(\bmod \mathfrak{a}) \in R \quad(i=1, \cdots, n)
$$

then $\mathfrak{p}^{m}$ is generated by $\left\{\delta_{1}^{v_{1}} \cdots \delta_{n}^{v_{n}} / \sum_{i=1}^{n} v_{i}=m\right\}$ and $\sum_{i=1}^{n} x_{i j} \cdot \delta_{i}=0$ for $1 \leq j \leq n+m$.

So if we define $\pi: \underline{\operatorname{Hom}}_{A}\left(A_{m+1}^{X}, A\right) \rightarrow \mathfrak{p}^{m}(n(n+m))$ by $\pi\left(\alpha^{*}\left(v_{1}, \cdots, v_{n}\right)\right)$ $=\delta_{1}^{v_{1}} \cdots \delta_{n}^{v_{n}}$, then $\pi$ is a surjective homomorphism of degree 0 and it follows from (2) that

$$
\begin{gathered}
\pi \cdot d_{m}^{*}\left(\beta^{*}\left(j ; w_{1}, \cdots, w_{n}\right)\right)=(-1)^{j+1} \sum_{q=1}^{n} x_{q j} \delta_{1}^{w_{1}} \cdots \delta_{q}^{w_{q}+1} \cdots \delta_{n}^{w_{n}} \\
=(-1)^{j+1}\left(\delta_{1}^{w_{1}} \cdots \delta_{n}^{w_{n}}\right) \sum_{q=1}^{n} x_{q j} \delta_{q}=0 .
\end{gathered}
$$

Hence the sequence;

$$
\underline{\operatorname{Hom}}_{A}\left(A_{m}^{X}, A\right) \underset{d_{m}^{*}}{\longrightarrow} \underline{\operatorname{Hom}}_{A}\left(A_{m+1}^{X}, A\right) \underset{\pi}{\longrightarrow} \mathfrak{p}^{m}(n(m+1)) \longrightarrow 0
$$

is a complex in $M_{H}(A)$ and $\pi$ is a surjection. Then the sequence (1) and (3) induce the surjective homomorphism $\psi: \underline{K}_{R}(n(n+m)) \rightarrow \mathfrak{p}^{m}(n(m+1))$. Here $\psi$ must be an isomorphism since $\underline{K}_{R}(n(n+m))$, as well as $\mathfrak{p}^{m}(n(m+1))$, is a fractional ideal of the integral domain $R$ by Lemma 1 . Hence we have $\underline{K}_{R}=\mathfrak{p}^{m}(-n(n-1))$.

(Q.E.D.)

Remark [Bruns]. In the theorem, $\mathfrak{p}$ is a prime ideal of height 1 , and the divisor class group $\mathrm{Cl}(R)$ of $R$ is a free abelian group generated by a single element $\operatorname{cl}(\mathfrak{p})$-the class of $\mathfrak{p}$.

Now let $\bar{f} \neq 0 \in R$ be an arbitrary homogeneous element of degree $n(n-1)$, then the theorem says that $\underline{K}_{R}$ is isomorphic to $\mathfrak{p}^{m} \cdot \bar{f}$, so the ring $R / p^{m} \bar{f}$ must be Gorenstein by Lemma 2. Hence if we choose the ideal $I$ of $A$ so that;

$$
A / I \cong R / \mathfrak{p}^{m} \bar{f}
$$

then $I$ is a Gorenstein ideal of height $(m+2)$. [Lemmas 2 and 3]

In case $m=1$, we can write down the resolution of $I$.

Proposition. Suppose $m=1$ in addition to the situation above. Then I has the following free resolution: 


$$
\begin{aligned}
& 0 \longrightarrow A\left(-n^{2}-n\right) \longrightarrow A(-n-1)^{n} \oplus A\left(-n^{2}\right)^{n+1} \\
& {\left[f \delta_{1}, \cdots, f \delta_{n}, \varepsilon_{1}, \cdots, \varepsilon_{n+1}\right]} \\
& \longrightarrow A\left(-n^{2}+1\right)^{n} \oplus A(-n)^{n+1} \longrightarrow A \longrightarrow A / I \longrightarrow 0 \\
& M \\
& \left(\begin{array}{c}
f \delta_{1} \\
\vdots \\
f \delta_{n} \\
\varepsilon_{1} \\
\vdots \\
\varepsilon_{n+1}
\end{array}\right]
\end{aligned}
$$

where

$$
\begin{aligned}
& \delta_{j}=(-1)^{j+1} \operatorname{det} Y_{j} \quad(1 \leq j \leq n) \\
& \varepsilon_{i}=(-1)^{i+1} \operatorname{det} X_{i} \quad(1 \leq i \leq n+1)
\end{aligned}
$$

$Y_{j}\left(\right.$ resp. $X_{i}$ ) is the matrix obtained from $Y$ (resp. $X$ ) by deleting its $j$-th row (resp. $i$-th column).

$f$ is an element of $A$ such that $f(\bmod , \mathfrak{a})=\bar{f} \in R$.

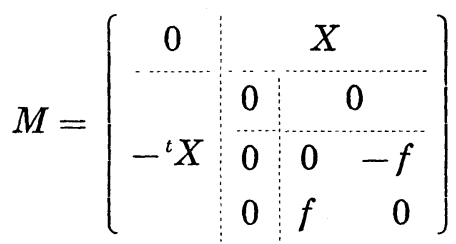

Proof. Easy calculation shows $M\left(\begin{array}{c}f \delta_{1} \\ \vdots \\ f \delta_{n} \\ \varepsilon_{1} \\ \vdots \\ \varepsilon_{n+1}\end{array}\right)=0$, that is, each row of $M$

is a relation among the generators $f \delta_{1}, \cdots, f \delta_{n}, \varepsilon_{1}, \cdots, \varepsilon_{n+1}$ of $I$. And moreover it is obvious that these relations are independent.

Since $I$ is a Gorenstein ideal of height 3 , the minimal number of generators of $I$ is equal to the number of relations among them. It follows from this fact that the rows of $M$ give all the relations among $f \delta_{1}, \cdots, f \delta_{n}, \varepsilon_{1}, \cdots, \varepsilon_{n+1}$ and $I$ is minimally generated by $f \delta_{1}, \cdots, f \delta_{n}$, $\varepsilon_{1}, \cdots, \varepsilon_{n+1}$. So the following is exact; 


$$
\begin{gathered}
A(-n-1)^{n} \oplus A\left(-n^{2}\right)^{n+1} \longrightarrow A\left(-n^{2}+1\right)^{n} \oplus A(-n)^{n+1} \longrightarrow A \longrightarrow A / I \longrightarrow 0 \\
\longrightarrow \\
{\left[\begin{array}{c}
f \delta_{1} \\
\vdots \\
f \delta_{n} \\
\varepsilon_{1} \\
\vdots \\
\varepsilon_{n+1}
\end{array}\right]}
\end{gathered}
$$

where the exactness is preserved by applying the functor $\operatorname{Hom}_{A}(, A)$ to this sequence, for $I$ is a perfect ideal of height 3, i.e. the sequence;

$$
\begin{gathered}
0 \longrightarrow A \\
{\left[f \delta_{1}, \cdots, f \delta_{n}, \varepsilon_{1}, \cdots, \varepsilon_{n+1}\right]}
\end{gathered}
$$

is exact. Hence we can replace ${ }^{t} M$ by $M$ for ${ }^{t} M=-M$. So we get the proposition.

(Q.E.D.)

\section{REFERENCES}

[B] W. Bruns, Die Divisorenklassengruppe der Restklassenringe von Polynomringen nach Determinantenidealen, Rev. Roum. Math. Pures et Appl. Bucarest XX No. 10 (1975), 1109-1111.

[E-N] J. A. Eagon and D. G. Northcott, Ideals defined by matrices and a certain complex associated with them, Proc. Roy. Soc. London Ser. A296 (1962), 188-204.

[G-W] S. Goto and K. Watanabe, On graded rings I, J. Math. Soc. Japan, 30 No. 2 (1978), 179-213.

[H-K] J. Herzog and E. Kunz, Der kanonische Modul eines Cohen-Macaulay Rings, Lecture Notes in Math. No. 238 Springer (1971).

[H-E] M. Hochster and J. A. Eagon, Cohen-Macaulay rings, invariant theory, and the generic perfection of determinantal loci, Amer. J. Math. 93 (1971), 1020-1058.

Nagoya University 